\title{
Avaliação reológica da hidroxiapatita
}

\section{(Rheological evaluation of hydroxyapatite)}

\author{
N. K. Porsani ${ }^{1}$, V. Trombini ${ }^{1}$, P. A. Ana ${ }^{1}$, L. F. G. Setz ${ }^{1}$ \\ ${ }^{I}$ Universidade Federal do ABC, Centro de Engenharia, Modelagem e Ciências Sociais Aplicadas, \\ Av. Estados 5001, 09210-580, Santo André, SP, Brasil
}

\begin{abstract}
Resumo
Os biomateriais, como a hidroxiapatita (HA), podem apresentar diferentes taxas de reabsorção no organismo em função da patologia a ser tratada e são dependentes das propriedades físico-químicas do biomaterial, onde a condição superficial é de fundamental importância. Neste trabalho, buscou-se desenvolver um biomaterial à base de $\mathrm{HA}$ com o silicato de sódio $\left(\mathrm{Na}_{2} \mathrm{SiO}_{3}\right)$ como dispersante e com potencial de formação de biovidro na superfície, além disso, conhecer as condições reológicas para se obter produtos adequados por conformação a úmido, via processamento coloidal. Caracterizou-se o material em termos da forma das partículas, observadas em microscópio, e área superficial específica. As medidas reológicas das suspensões utilizadas foram realizadas em reômetro rotacional. Observou-se a possibilidade de produção do biomaterial proposto, conformado em moldes porosos, através de suspensões com $2,0 \%$ em massa de silicato de sódio trabalhando-se preferencialmente em taxas de cisalhamento abaixo de $200 \mathrm{~s}^{-1}$.
\end{abstract}

Palavras-chave: reologia, hidroxiapatita, biomateriais, colagem em moldes porosos.

\begin{abstract}
Biomaterials, such as hydroxyapatite (HA), may present different rates of reabsorption in the organism depending on the pathology to be treated. The organism reabsorption rate depends on the physicochemical properties of the biomaterial, where the superficial condition has a fundamental importance. The aim of this work was to develop an HA-based biomaterial with sodium silicate $\left(\mathrm{Na}_{2} \mathrm{SiO}_{3}\right)$ as a dispersant and with the potential to form bioglass on the surface, in addition, to know the rheological conditions to obtain suitable products by wet conformation, by colloidal processing route. The material used was characterized in terms of particle shape, observed in a microscope, and specific surface area. The suspensions rheological measurements were performed in a rotational rheometer. It was observed the possibility of production of the proposed biomaterial shaped by slip casting, using suspensions with $2.0 \mathrm{wt} \%$ of sodium silicate, preferably working at shear rates below $200 \mathrm{~s}^{-1}$.
\end{abstract}

Keywords: rheology, hydroxyapatite, biomaterials, slip casting.

\section{INTRODUÇÃO}

No estudo dos biomateriais para tratamentos de enfermidades ósseas, sempre existe a preocupação com a osseointegração, ou seja, a interação bioquímica entre o tecido ósseo e a superfície do implante empregado, de modo a promover a reconstituição óssea [1]. A utilização de materiais que atendam essas demandas é imprescindível e, assim, os materiais à base de fosfato de cálcio, por apresentarem excelente biocompatibilidade, são os materiais sintéticos mais empregados em enxertia óssea [2,3]. Dentre os demais componentes da estrutura óssea, além dos compostos de cálcio e fósforo, encontram-se íons como bicarbonato, magnésio, potássio, sódio e citratos em pequenas quantidades, além de elementos celulares, proteínas como colágeno tipo I e proteoglicanas, além de outros tecidos, vasos e fluidos, com distribuição e morfologia específica para cada região do corpo humano [4]. Dentre os diversos materiais à base de fosfato de cálcio, a hidroxiapatita (HA) apresenta-se como material de referência para substituição e regeneração óssea, pois, além da sua similaridade com a parte mineral de ossos e dentes - compreende cerca de 55\% da composição dos ossos,
96\% da composição do esmalte dentário e $70 \%$ da dentina, exibe excelente biocompatibilidade, osteocondutividade e bioatividade capaz de permitir a proliferação de células ósseas, favorecendo os processos de regeneração óssea [5]. Além do mais, por permitir substituições aniônicas e catiônicas com facilidade, atua também como um sistema regulador de diferentes íons nos líquidos corpóreos. Essa característica é também responsável pela crescente utilização da HA na área ambiental para o tratamento de solos e águas contaminados [6]. Outra propriedade da HA é a adsorção de proteínas em sua superfície [5].

Muitos trabalhos têm evidenciado a regeneração óssea empregando-se HA, majoritariamente sintetizada empregando-se outros dispersantes que não o silicato de sódio. O silicato de sódio apresenta-se atrativo para a síntese, pois se trata de um material disponível comercialmente e de menor custo, além de apresentar a possibilidade de formação de biovidro. Tais características fazem com que o silicato de sódio seja vantajoso para produção de um biomaterial mais barato e com maior possibilidade de osseointegração e osseoindução. Na literatura, contudo, não há trabalhos que empregaram o silicato de sódio como dispersante 
para a HA, o que motivou a realização do presente estudo. Além disso, o conhecimento das propriedades reológicas dos biomateriais cerâmicos, mais especificamente da hidroxiapatita, possibilita obter elementos de enxertia óssea adequados, com as características desejadas aos processos que proporcionem a biointegração [7]. Entre os processos de conformação a úmido que podem ser empregados, a colagem em moldes porosos (geralmente gesso), afigurase adequada, pois é uma técnica relativamente simples que possibilita a conformação de peças com geometrias complexas. Esta técnica baseia-se no princípio de se verter uma suspensão estável em molde seco e poroso a qual, por meio de capilaridade, a água é absorvida sendo depositada nas paredes do molde uma camada uniforme do material. Quando a camada atinge as dimensões desejadas e tornase rígida o suficiente para suportar o próprio peso, ela é então removida. Por vezes produzem-se também materiais maciços por esta técnica $[8,9]$. Diante do exposto, este trabalho busca o desenvolvimento e a caracterização de um novo biomaterial, com maior possibilidade de bioatividade e de menor custo, o que é relevante para a realidade atual.

\section{MATERIAIS E MÉTODOS}

O material utilizado no estudo foi a hidroxiapatita comercial (HA, P.A., CAAL, Brasil) e para ele determinouse a área de superfície específica (Quantachrome Instr., Nova 2200e, EUA) e observou-se a forma das partículas por meio de microscópio eletrônico de varredura (FEI, Quanta 250, EUA). A estabilidade da hidroxiapatita (curva de potencial zeta) foi executada no zetâmetro Zeta PALS (Brookhaven Instr., EUA). Prepararam-se, para isso, suspensões com concentrações de $0,01 \%$ em volume de sólidos, utilizandose água ultrapura (Milli-Q, Merck Millipore, Alemanha) ajustando-se o $\mathrm{pH}$ com quantidades adequadas de $\mathrm{KOH}$ e $\mathrm{HCl}$ $\left(10^{-3} \mathrm{M}\right)[10,11]$. As suspensões estudadas foram produzidas utilizando-se água destilada e o dispersante, silicato de sódio ( $\mathrm{Na}_{2} \mathrm{SiO}_{3}$, Casa do Ceramista, Brasil), foi ajustado por meio de curva de defloculação em uma suspensão contendo $50 \%$ em massa de sólidos. Este dispersante $\left(\mathrm{Na}_{2} \mathrm{SiO}_{3}\right)$ foi escolhido por ser facilmente encontrado no mercado, uma vez que é utilizado em escala na produção de 'barbotinas' em indústrias cerâmicas tradicionais $[8,12,13]$, além de fornecer $\mathrm{SiO}_{2}$ ao material estudado que pode contribuir com a formação de fase vítrea (biovidro) nas superfícies, melhorando os efeitos osteoindutores da hidroxiapatita [1416]. Esta última possibilidade, a da formação de biovidro na superfície das partículas de HA, será estudada em futuros trabalhos.

O período de homogeneização das suspensões, após determinação da concentração de dispersante, foi de $24 \mathrm{~h}$ em moinho de bolas para adequada homogeneidade [17]. A curva de defloculação foi realizada em viscosímetro analógico rotacional tipo Brookfield (Q860A, Quimis, Brasil). As demais medidas reológicas foram realizadas em reômetro rotacional (Viscotester IQ, Thermo Haake, Alemanha) utilizando cilindros concêntricos como sensor.
As curvas foram obtidas no modo de velocidade (taxa) controlada (controlled rate - CR) elevando-se de 0 até 1000 $\mathrm{s}^{-1}$, em $500 \mathrm{~s}$, permanecendo nessa velocidade por $60 \mathrm{~s}$ e retornando até $0 \mathrm{~s}^{-1}$ também em $500 \mathrm{~s}$. Todos os parâmetros reológicos, como os valores de tixotropia e os ajustes do modelo de Cross, foram extraídos a partir das curvas de fluxo obtidas no modo CR utilizando-se o software Haake RheoWin 4.63.0003. Para cada suspensão produzida, foram feitas colagens em moldes de gesso de pastilhas com 10 $\mathrm{mm}$ de diâmetro (aproximadamente) e $10 \mathrm{~mm}$ de espessura. Todas as peças conformadas foram secas em estufa a 100 ${ }^{\circ} \mathrm{C}$ por $24 \mathrm{~h}$. As densidades a verde foram determinadas geometricamente.

\section{RESULTADOS E DISCUSSÃO}

Estabilidade das partículas: apresentando área de superfície específica de $44,51 \mathrm{~m}^{2} \cdot \mathrm{g}^{-1}$, o aspecto de forma das partículas da hidroxiapatita (HA) comercial é apresentado na Fig. 1. Observou-se, por meio de microscópio eletrônico de varredura, que o pó de hidroxiapatita apresentava formas lamelares (Fig. 1), com variação nas dimensões das partículas em torno de $3,0 \mu \mathrm{m}$. O tamanho e a forma das partículas são importantes e influenciam diretamente o comportamento reológico das suspensões concentradas [18-20], pois, em suspensões com concentrações elevadas de sólidos ( $>65 \%$ em massa) [21], sob cisalhamento $\left(>100 \mathrm{~s}^{-1}\right)$, a viscosidade do sistema pode ser elevada pelo efeito da dilatância (espessamento frente ao fluxo) devido a mudanças no fator de empacotamento [18].

AFig. 2 apresenta a curva de potencial zeta do HA em água. Conforme observado em [22], o valor do ponto isoelétrico ocorreu a um valor próximo de $\mathrm{pH} 9 \mathrm{e}$, portanto, considerando

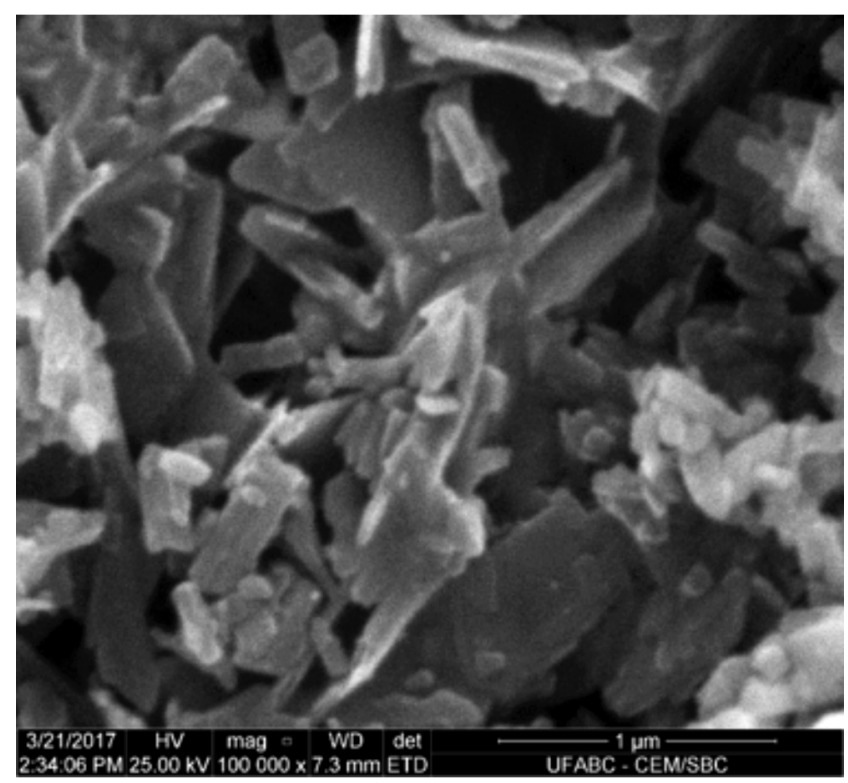

Figura 1: Micrografia obtida por microscopia eletrônica de varredura ilustrando o aspecto lamelar e aglomerado das partículas de HA.

[Figure 1: SEM micrograph showing HA agglomerate and lamellar particle aspect.] 
o processamento cerâmico, deve-se trabalhar o mais afastado possível deste valor de $\mathrm{pH}$. Para que seja possível alcançar a estabilidade eletrostática em meio aquoso, de acordo com a curva apresentada, é necessário que se trabalhe em potenciais acima de $120 \mathrm{l} \mathrm{mV}$, ou seja, acima de $\mathrm{pH}$ 10,0 [20, $23,24]$. Entretanto, devido ao fato de que estas medidas foram realizadas em suspensões com baixa concentração de sólidos ( $\sim 0,01 \%$ vol) a interação entre partículas é muito baixa e ao se produzir suspensões com altas concentrações de sólidos esta situação se inverte, havendo forte interação entre as partículas do meio. Deste modo, por vezes, faz-se necessário o uso de aditivos que promovam, além da estabilização das partículas no meio, a formação de barreiras mais eficientes do que a estabilização eletrostática, para minimizar os efeitos das forças de atração interparticulares [20, 23, 25]. Este comportamento pode ser obtido pela utilização de dispersantes, no caso, o silicato de sódio, cuja melhor concentração é apresentada na Fig. 3, através da curva de defloculação.

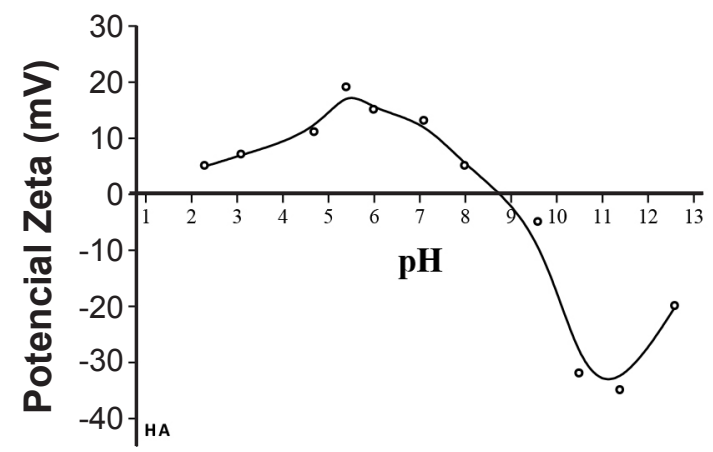

Figura 2: Curva de potencial zeta versus $\mathrm{pH}$ para HA.

[Figure 2: HA zeta potential versus pH curve.]

Preparo de suspensões: a curva de defloculação, ou seja, a determinação da quantidade de dispersante necessária à estabilização das suspensões de HA, foi obtida utilizando-se silicato de sódio, em suspensões com concentração de 50\% em massa. Na curva de defloculação, apresentada na Fig. 3, observa-se uma maior redução no valor da viscosidade para adições de $0,5 \%$ em massa de silicato de sódio, a partir da qual há uma redução dos valores de viscosidade menos intensa. Isso indica que a condição de estabilidade está sendo satisfeita, ou seja, as forças superficiais de repulsão já estão atuando no sentido de estabilizar as partículas no meio. Porém, a menor viscosidade só é alcançada com a concentração de 1,5\% em massa de silicato de sódio. A partir dessa concentração, a viscosidade apresenta um valor quase constante, mas com uma leve tendência a voltar a subir, que fica mais nítida quando a concentração de dispersante passa de $2,5 \%$. Sendo assim, na sequência do estudo, as concentrações de 1,5 e 2,0\% de silicato de sódio foram utilizadas. Todas as suspensões foram produzidas utilizando água destilada e submetidas à agitação em moinho de bolas por $24 \mathrm{~h}$ para garantir uma boa homogeneidade das suspensões [11, 21]. Todas as medidas realizadas apresentaram desvio padrão nas medidas de aproximadamente 5\%, que é o erro do reômetro, indicado pelo fabricante.

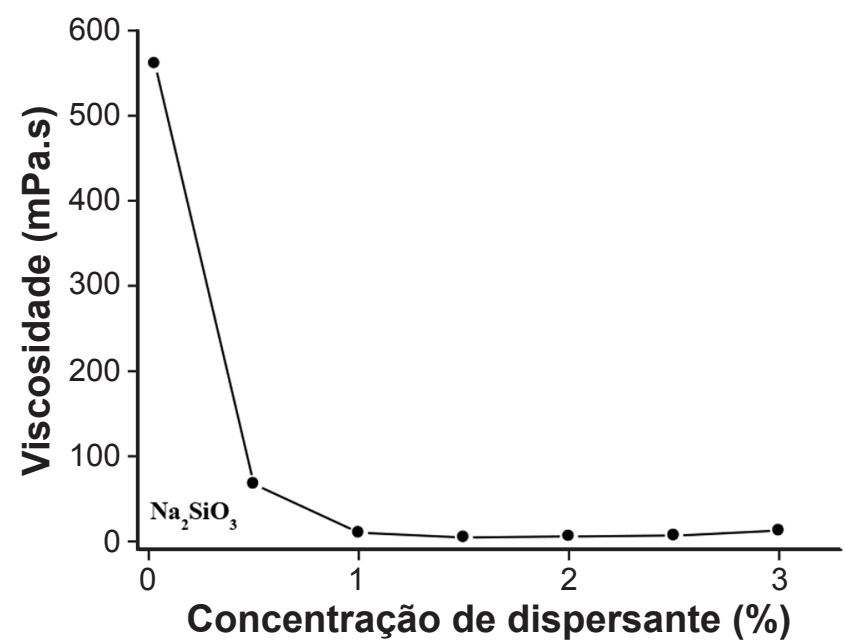

Figura 3: Curva de defloculação de suspensões aquosas de HA, com silicato de sódio como dispersante.

[Figure 3: HA aqueous deflocculation curve with sodium silicate as dispersant.]

Na Tabela I são apresentadas as análises reológicas, realizadas no viscosímetro rotacional, das suspensões utilizadas para elaboração da curva de defloculação. Os valores dos índices tixotrópicos foram determinados pelas medidas das viscosidades (valores médios) logo depois de colocadas no equipamento $\left(\eta_{\mathrm{t} 0}\right)$ e após dez minutos $\left(\eta_{\mathrm{t} 10}\right)$. Os valores indicaram uma melhoria no comportamento da suspensão com 1,5\% de silicato de sódio, acompanhando o resultado da estabilidade observado na Fig. 3. Os índices tixotrópicos são particularmente úteis, pois indicam a ocorrência do fenômeno de tixotropia, porém não se trata de uma avaliação reológica propriamente dita, mas sim de uma inferência de valores práticos que indicam, neste caso, a linearidade da suspensão preparada com o tempo, ou seja, sua uniformidade frente ao fluxo com o tempo [26]. Neste caso, conforme esperado, na condição de maior estabilidade (menor viscosidade da suspensão), ou seja, com a concentração de $1,5 \%$ de silicato de sódio, a suspensão

Tabela I - Parâmetros reológicos com diferentes concentrações de dispersante.

[Table I - Rheological parameters with different dispersant content.]

\begin{tabular}{cccc}
\hline $\begin{array}{c}\mathrm{SiNa} \\
(\% \mathrm{~m})\end{array}$ & $\begin{array}{c}\eta_{\mathrm{t} 0} \\
(\mathrm{mPa} . \mathrm{s})\end{array}$ & $\begin{array}{c}\eta_{\mathrm{t} 10} \\
(\mathrm{mPa} . \mathrm{s})\end{array}$ & $\begin{array}{c}\text { Índice } \\
\text { tixotrópico }\end{array}$ \\
\hline 0,0 & 561,1 & 615,3 & 0,91 \\
0,5 & 67,6 & 92,6 & 0,73 \\
1,0 & 9,9 & 20,5 & 0,48 \\
1,5 & 4,6 & 15,8 & 0,29 \\
2,0 & 5,8 & 16,0 & 0,36 \\
2,5 & 6,7 & 15,0 & 0,45 \\
3,0 & 12,5 & 18,6 & 0,67 \\
\hline Nota: $\eta_{t 0}$ e $\eta_{t 10}$ correspondem às viscosidades nos instantes 0 e 10 min.
\end{tabular}


apresentou menor variação da propriedade com tempo. Os valores dos índices tixotrópicos foram todos inferiores a 1,0 , indicando que as suspensões estudadas são reopéxicas, ou seja, as viscosidades das suspensões aumentam com tempo para uma mesma rotação dos spindles. Nos viscosímetros rotacionais tipo Brookfield, como não existe um recipiente com uma geometria específica para a medição das viscosidades, não é possível calcular as taxas de cisalhamento, entretanto, há uma relação direta com a rotação do equipamento [18].

As curvas de fluxo para diferentes concentrações de sólidos, com concentrações de silicato de sódio de 1,5 e
2,0\% em massa, apresentadas respectivamente nas Figs. 4a e 4b, mostraram um significativo aumento na resistência ao fluxo (viscosidade) das suspensões com o aumento na concentração de sólidos e foi de acordo com o esperado. De maneira geral, no processamento cerâmico, dentro dos parâmetros de cada processo de conformação busca-se sempre a maior concentração de sólidos possível de modo a minimizar os problemas na etapa de secagem [8, 12]. Observou-se também que todas as suspensões apresentaram comportamentos predominantemente fluidificantes frente ao fluxo (pseudoplásticos), além da reopexia, que é a histerese (área) existente entre as curvas de subida e descida para cada
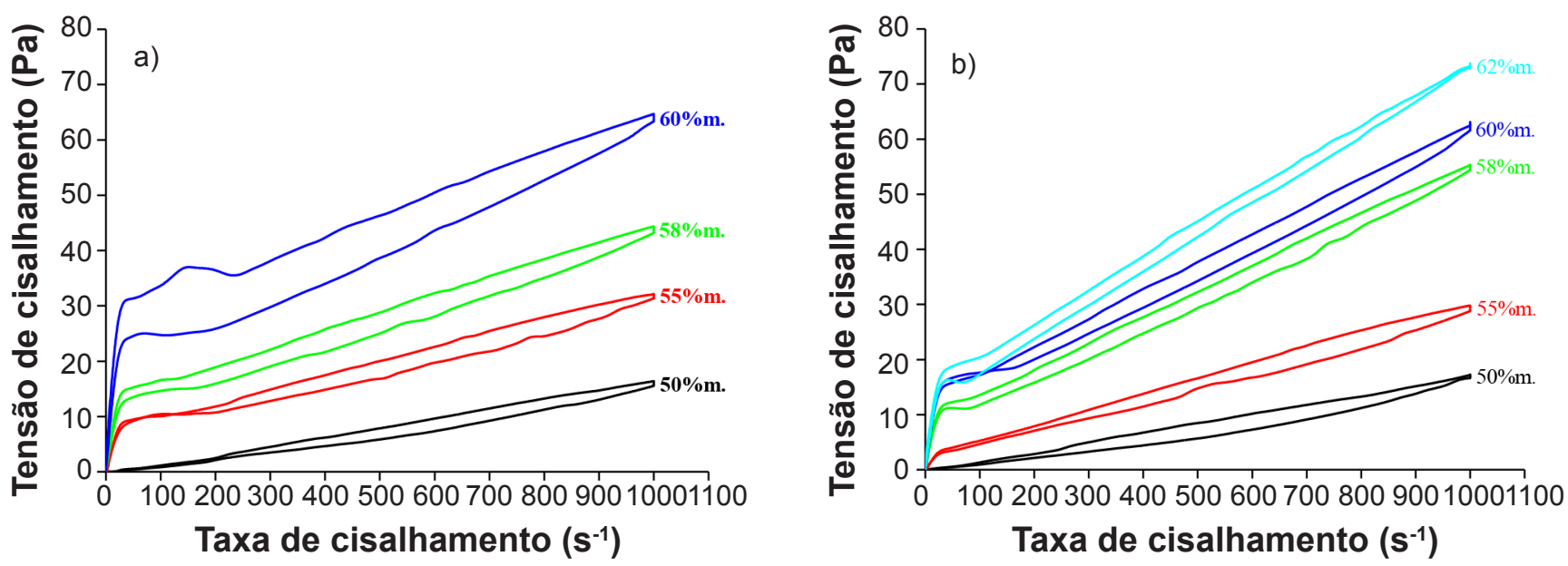

Figura 4: Curvas de fluxo em função da concentração de sólidos, com 1,5\% (a) e 2,0\% (b) em massa de silicato de sódio como dispersante. [Figure 4: Viscosity curves with varying solid contents and $1.5 \mathrm{wt} \%$ (a) and $2.0 \mathrm{wt} \%$ (b) of sodium silicate as dispersant.]

Tabela II - Propriedades reológicas de suspensões preparadas com 1,5\% em massa de silicato de sódio. [Table II - Rheological properties for suspensions prepared with $1.5 \mathrm{wt} \%$ sodium silicate.]

\begin{tabular}{cccccc}
\hline $\begin{array}{c}\text { Conc. sólidos } \\
(\% \mathrm{~m})\end{array}$ & $\begin{array}{c}\text { Conc. sólidos } \\
(\% \text { vol })\end{array}$ & $\begin{array}{c}\text { Parâmetros do modelo Cross } \\
\text { Viscosidade a } \\
0 \mathrm{~s} \mathrm{~s}^{-1}(\mathrm{~Pa} . \mathrm{s})\end{array}$ & $\begin{array}{c}\text { Viscosidade } \\
\text { limite }(\mathrm{Pa} . \mathrm{s})\end{array}$ & $\begin{array}{c}\text { Tixotropia } \\
\left(\mathrm{Pa} . \mathrm{s}^{-1}\right)\end{array}$ & $\begin{array}{c}\text { Viscosidade a } \\
10 \mathrm{~s}^{-1}(\mathrm{~Pa} . \mathrm{s})\end{array}$ \\
\hline 50,0 & 24,0 & 0,0166 & 0,0120 & $-1401,0$ & 0,007 \\
55,0 & 27,9 & 0,4455 & 0,0286 & -21730 & 0,373 \\
58,0 & 30,4 & 1,1770 & 0,0377 & $-3061,0$ & 0,599 \\
60,0 & 32,2 & 2,8890 & 0,0538 & $-7041,0$ & 1,141 \\
\hline
\end{tabular}

Tabela III - Propriedades reológicas de suspensões preparadas com 2,0\% em massa de silicato de sódio. [Table III - Rheological properties for suspensions prepared with $2.0 \mathrm{wt} \%$ sodium silicate.]

\begin{tabular}{cccccc}
\hline $\begin{array}{c}\text { Conc. sólidos } \\
(\% \mathrm{~m})\end{array}$ & $\begin{array}{c}\text { Conc. sólidos } \\
(\% \mathrm{vol})\end{array}$ & $\begin{array}{c}\text { Parâmetros do modelo Cross } \\
\text { Viscosidade a } \\
0 \mathrm{~s}^{-1}(\mathrm{~Pa} . \mathrm{s})\end{array}$ & $\begin{array}{c}\text { Viscosidade } \\
\text { limite }(\mathrm{Pa} . \mathrm{s})\end{array}$ & $\begin{array}{c}\text { Tixotropia } \\
\left(\mathrm{Pa}_{\mathrm{s}}{ }^{-1}\right)\end{array}$ & $\begin{array}{c}\text { Viscosidade a } \\
10 \mathrm{~s}^{-1}(\mathrm{~Pa} . \mathrm{s})\end{array}$ \\
\hline 50,0 & 24,0 & 0,0453 & 0,0106 & $-905,6$ & 0,008 \\
55,0 & 27,9 & 0,1552 & 0,0272 & $-2024,0$ & 0,143 \\
58,0 & 30,4 & 1,5510 & 0,0399 & $-2500,0$ & 0,632 \\
60,0 & 32,2 & 2,3540 & 0,0516 & $-3077,0$ & 0,752 \\
62,0 & 34,0 & 37,0800 & 0,0637 & $-2981,0$ & 0,959 \\
\hline
\end{tabular}




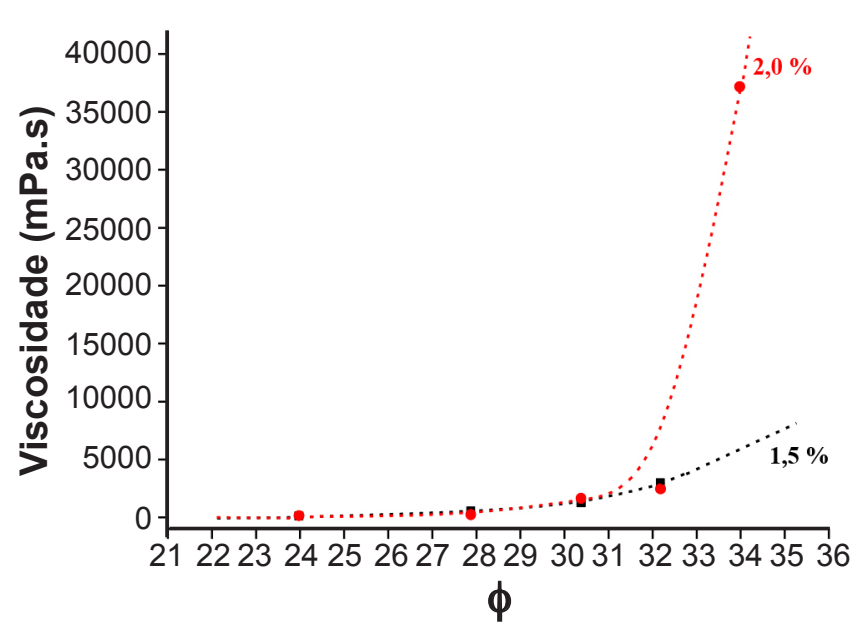

Figura 5: Curva de viscosidade versus concentração de sólidos $(\phi)$, de acordo com modelo de Krieger-Dougherty.

[Figure 5: Viscosity curves versus solid concentration ( $\phi)$, adjusted by Krieger-Dougherty model.]

uma das suspensões estudadas. Este comportamento fica mais acentuado com o aumento na concentração de sólidos. De uma maneira geral, no processamento cerâmico, buscase sempre o afastamento da última condição [18]; por outro lado, como ela ocorre em todas as suspensões estudadas, o que pode ser feito é se trabalhar em menores concentrações de sólidos, neste caso abaixo de $60 \%$ em massa, pois a ocorrência da reopexia, devido ao menor grau de choque entre as partículas suspensas, é menor. Observou-se também que em velocidades de cisalhamento abaixo de $200 \mathrm{~s}^{-1}$ a histerese quase inexistiu para as suspensões contendo 50 e $55 \%$ em massa de sólidos e foi pequena acima destas concentrações, sendo, portanto, mais um bom indicativo das melhores condições para se trabalhar com estas suspensões.

As análises reológicas de todas as suspensões estudadas, com 1,5\% em massa de silicato de sódio, são apresentadas na Tabela II. Os valores negativos de tixotropia indicam que todas as suspensões são reopéxicas, confirmando o comportamento observado na Tabela I. Todas as curvas foram ajustadas pelo modelo reológico de Cross, que permite predizer convenientemente a forma geral das curvas de fluxo, diferenciando as regiões de baixa e alta taxas de cisalhamento, ou seja, os valores de viscosidade limite extrapolados à taxa de cisalhamento zero $\left(\eta_{0}\right)$ e à taxa de cisalhamento infinita $\left(\eta_{\infty}\right)$, o que fornece informações importantes e precisas do comportamento nas condições de altas e muito baixas velocidades de cisalhamento, próximas às condições de repouso [20]. Ademais das melhores condições para se trabalhar com as suspensões abaixo de 200 $\mathrm{s}^{-1}$, os valores das viscosidades à taxa de cisalhamento $10 \mathrm{~s}^{-1}$ atende os requisitos para o processo de colagem em moldes porosos [8]. Por outro lado, após o processo de moagem, devido aos processos de desaglomeração envolvidos, além da homogeneização, decidiu-se considerar no preparo das suspensões a concentração de 2,0\% de silicato de sódio de forma a garantir uma cobertura total das partículas em suspensão $[11,20]$. Os resultados destas análises são apresentados na Fig. 4b, onde se observa um comportamento semelhante aos encontrados quando da utilização de 1,5\% em massa (Fig. 4a), porém, nesta nova condição, observouse uma menor viscosidade para as mesmas concentrações de sólidos, além de menores valores de tixotropia (área existente entre as curvas de fluxo na subida e na descida), o que justifica a escolha de 2,0\% de silicato de sódio como dispersante. As análises destas curvas são apresentadas na Tabela III.

Na Fig. 5 são apresentadas as curvas das viscosidades limites em função da fração de volume de sólidos, ajustadas de acordo com o modelo de Krieger-Dougherty, para suspensões contendo 1,5 e 2,0\% de silicato de sódio. Observou-se um aumento na viscosidade dinâmica com o aumento na concentração de sólidos, até uma concentração máxima de aproximadamente $33 \%$ em volume, para $2,0 \%$ de dispersante, muita próxima à concentração utilizada neste estudo. A partir desta concentração, a suspensão perde as condições de fluxo necessárias à conformação em moldes porosos. Nas suspensões contendo 1,5\% de silicato de sódio, atingiram-se valores de viscosidades, nas mesmas concentrações de sólidos, ligeiramente menores, indicando uma melhor trabalhabilidade das suspensões nestas condições; porém, para adições acima de 58\% em massa, as suspensões encontraram-se no limiar das viscosidades adequadas para colagem em moldes porosos $[8,19,20]$. Adições superiores a 2,0\% mostraram-se na prática inadequadas para a produção de suspensões de HA, confirmando os resultados observados na Fig. 3.

Colagem em moldes porosos: as densidades das pastilhas conformadas por colagem em moldes porosos de gesso [27]

Tabela IV - Densidades das pastilhas conformadas por colagem em moldes porosos.

[Table IV - Samples densities shaped by slip-casting.]

\begin{tabular}{cccc}
\hline $\begin{array}{c}\text { Conc. sólidos } \\
(\% \mathrm{~m})\end{array}$ & $\begin{array}{c}\text { Conc. sólidos } \\
(\% \mathrm{vol})\end{array}$ & $\begin{array}{c}\text { Densidade relativa a verde, } \\
1,5 \% \mathrm{~m} \mathrm{Na}_{2} \mathrm{SiO}_{3}(\%)\end{array}$ & $\begin{array}{c}\text { Densidade relativa a verde, } \\
2,0 \% \mathrm{ma}_{2} \mathrm{SiO}_{3}(\%)\end{array}$ \\
\hline 50,0 & 24,0 & $42,6 \pm 2,0$ & $48,4 \pm 1,2$ \\
55,0 & 27,9 & $42,9 \pm 2,3$ & $48,1 \pm 2,5$ \\
58,0 & 30,4 & $49,8 \pm 1,6$ & $48,3 \pm 1,2$ \\
60,0 & 32,2 & $46,3 \pm 2,8$ & $47,4 \pm 3,0$ \\
62,0 & 34,0 & - & $46,9 \pm 1,9$ \\
\hline
\end{tabular}


são apresentadas na Tabela IV. Os resultados indicaram proximidade em todos os valores das densidades a verde independente das concentrações de sólidos quando mantida a mesma concentração de dispersante, indicando uma boa uniformidade em todas as suspensões produzidas, porém com maior concentração de dispersante observou-se uma maior uniformidade na distribuição de valores, indicando que houve maior homogeneidade quando se empregou $2,0 \%$ em massa de silicato de sódio. Uma vez que os valores de densidades foram próximos, a diferença que há variandose a quantidade de sólidos é que a velocidade de formação das camadas de material é mais rápida quanto maior for a concentração de sólidos das suspensões. Os valores das densidades a verde indicaram ainda que os graus de porosidade a serem obtidos após a sinterização devem ser adequados às demandas para elementos de enxertia óssea [28]. A colagem da suspensão com concentração de $62 \%$ em massa não foi possível com 1,5\% de silicato de sódio, pois não foi possível produzi-la de forma que mantivesse as características de uma suspensão [20].

\section{CONCLUSÕES}

A produção de suspensões aquosas de hidroxiapatita é possível com a utilização de $2,0 \%$ em massa de silicato de sódio como dispersante, que devem ser utilizadas preferencialmente em velocidades de cisalhamento abaixo de $200 \mathrm{~s}^{-1}$ para que possam ser conformadas em moldes porosos. Para adequada viabilidade dos materiais estudados para utilização em elementos de enxertia óssea, porém, estudos posteriores de biocompatibilidade são necessários.

\section{AGRADECIMENTOS}

Os autores agradecem o apoio da FAPESP (Processos $\mathrm{N}^{\circ}$ 2013/09329-5 e 2015/16859-6) e CNPq (420217/2016-9) e à Central Experimental Multiusuário (CEM) da UFABC.

\section{REFERÊNCIAS}

[1] T.D. Driskell, in: "Characterization and performance of calcium phosphate coatings for implants", Eds.: E. Horowitz, J.E. Parr, ASTM Int., Philadelphia (1994).

[2] S.S. Dalapicula, M.B. Conz, Rev. ImplantNews 5 (2008) 205.

[3] U. Almeida, "Análise e utilização de biomaterial confeccionado a partir das conchas de Crassostrea Gigas em defeito periodontal em ratos", Diss. Mestr., Un. Positivo, Curitiba (2010).

[4] L.C. Junqueira, J. Carneiro, P. Abrahamsohn, Histologia básica, 13ª Ed., Guanabara Koogan, Rio de Janeiro (2017) 568.
[5] C. Rey, C. Combes, C. Drouet, H. Sfihi, A. Barroug, Mater. Sci. Eng. C 27 (2007) 198.

[6] A.A. Campbell, Mater. Today 6 (2003) 26.

[7] H. Murata, in: "Polymerization", Ed. A.S. Gomes, Intech (2012) 403.

[8] J.S. Reed, Principles of ceramics processing, $2^{\text {nd }}$ Ed., John Wiley \& Sons, New York (1995).

[9] D.W. Richerson, Modern ceramic engineering: properties, processing, and use in design, $2^{\text {nd }} \mathrm{Ed}$., Marcel Dekker, 1992.

[10] L.F.G. Setz, S.R.H. Mello-Castanho, M.T. Colomer, R. Moreno, Solid State Ionics 180 (2009) 71.

[11] L.F.G. Setz, I. Santacruz, M.T. Colomer, S.R.H. MelloCastanho, R. Moreno, Mater. Res. Bull. 46 (2011) 983.

[12] T.A. Ring, Fundamentals of ceramic powder processing and synthesis, Academic Press, San Diego (1996).

[13] A.P.N. Oliveira, D. Hotza, Tecnologia de revestimentos cerâmicos, $2^{a}$ Ed., Edi. UFSC, Florianópolis (2015).

[14] B. Fan, X.H. Wang, H. Zhang, P. Gao, H. Zhang, X. Li, H. Huang, X. Xiao, D.J. Liu, Q. Lian, Z. Guo, Z. Wang, Mater. Des. 112 (2016) 67.

[15] Y. Wan, T. Cui, W. Li, C.C. Li, J. Xiao, Y.Zhu, D. Ji, G. Xiong, H. Luo, Mater. Des. 99 (2016) 521.

[16] F. Baino, S. Fiorilli, C. Vitale-Brovarone, Acta Biomater. 42 (2016) 18.

[17] L.F.G. Setz, "Processamento coloidal de cromito de lantânio", Tese Dr, Inst. Pesq. Energ. Nucl., S. Paulo (2009). [18] D. Dinger, Rheology for ceramists (2003).

[19] T.F. Tadros, Rheology of dispersions: principles and applications, Wiley-VCH Verlag, Weinheim (2010).

[20] R. Moreno, Reología de suspensiones cerámicas, Cons. Sup. Invest. Cient., Madrid (2005).

[21] L.F.G. Setz, A.C. Silva, S.C. Santos, S.R.H. MelloCastanho, M.R. Morelli, J. Eur. Ceram Soc. 33 (2013) 3211. [22] C. Ribeiro, "Obtenção e caracterização de viocerâmicas porosas a base de fosfatos de cálcio processadas com autilização de albumina", Tese Dr, Inst. Pesq. Energ. Nucl., S. Paulo (2008).

[23] J.N. Israelachvili, Intermolecular and surface forces, $2^{\text {nd }}$ Ed., Academic Press, Oxford (1992).

[24] R.J. Pugh, L. Bergström, Surface and colloid chemistry in advanced ceramics processing, Marcel Dekker, New York (1994).

[25] J.C. Berg, A introduction to interfaces \& colloids: the bridge to nanoscience, World Sci. Publ. (2010).

[26] D. Laba, Rheological properties of cosmetics and toiletries, Marcel Dekker, New York (1993).

[27] M. Spătaru, C. Ţaărdei, M.R. Nemţanu, F. Bogdan, Rev. Roum. Chim. 53 (2008) 955.

[28] C. Ribeiro, J.C. Bressiani, A.H.A. Bressiani, Mater. Res. 10 (2007) 307.

(Rec. 31/07/2017, Rev. 24/10/2017, 20/12/2017, Ac. 26/12/2017) 